\title{
FVTD Simulations of Archimedean Spiral Antennas on Thin Substrates in Planar and Conformal Configurations
}

\author{
Christophe Fumeaux*, Dirk Baumann, Rüdiger Vahldieck \\ Swiss Federal Institute of Technology, ETHZ, IFH, 8092 Zürich, Switzerland; \\ fumeaux@ifh.ee.ethz.ch
}

\begin{abstract}
This paper demonstrates the applicability of the Finite-Volume Time-Domain (FVTD) method to the simulation of two-arm substrate-supported Archimedean spiral antennas. The FVTD method uses an unstructured tetrahedral mesh to model precisely the fine spiral structures and the thin underlying substrate. Time-domain simulation results are presented to demonstrate the substrate influence on the current distribution on the metallic spiral arms. Application of the FVTD method to conformal spiral geometries is suggested in two further examples.
\end{abstract}

Keywords: FVTD, time-domain analysis, spiral antenna, conformal antennas

\section{Introduction}

Archimedean spiral antennas are widely used as circularly polarized broadband devices for airborne RF applications because of their simplicity, compactness and stable radiation characteristics over a large bandwidth. The Archimedean spiral antenna is characterized by a constant line width and therefore is not a truly frequencyindependent radiator. However, circular polarization and remarkably stable radiation patterns are achievable over bandwidths of more than one decade. The lowest operational frequency of the spiral is determined by its outer radius which defines the overall dimensions of the device. The upper frequency limit is set by the inner spiral radius, the center of which is the antenna feed point. The typical Archimedean spiral antenna exhibits narrow arms and a large number of windings, making it a challenging problem for any computational method. In the present paper, the Finite-Volume Time-Domain (FVTD) method is used to simulate the Archimedean spiral on a thin substrate. The FVTD technique benefits from its use of an unstructured mesh. This permits to approximate the curved spiral geometry with great accuracy and to adapt the cell size locally to resolve fine geometrical features of the device (spiral arms, thin substrate). Various spiral geometries have been modeled. The present paper first places the emphasis on the effect of the substrate and second, briefly demonstrates the application of the method to conformal geometries.

\section{Finite-Volume Time-Domain Model of the Spiral Antenna}

This section summarizes the relevant characteristics of the spiral simulation, showing in particular the spatial discretization of the modeled structure.

\section{a) The FVTD Method}

The FVTD method has been introduced at the end of the 80's [1] as a numerical technique to solve Maxwell's equations in an unstructured mesh. The method is based on integrations of a conservative form of Maxwell's curl equations over elementary cells (finite volumes). The FVTD formulation used in this investigation considers a flux-splitting cell-centered algorithm proposed by Bonnet [2]. A tetrahedral mesh provides the spatial discretization of the antenna.

\section{b) The Spiral Antenna}

An Archimedean spiral is defined in polar coordinates $(\rho, \phi)$ as $\rho=a_{0}\left(\phi+\phi_{0}\right)$, where $a_{0}$ defines the winding tightness and $\phi_{0}$ is the starting angle that determines the inner radius of the spiral. The modeled selfcomplementary spiral has an arm width of $d_{S}=0.25 \mathrm{~mm}$, an outer radius of $R=11.25 \mathrm{~mm}$, an inner radius of $r=0.25 \mathrm{~mm}$ and is supported by a substrate with a thickness of $h=0.254 \mathrm{~mm}$ (Figure 1). The dimensions of the spiral are challenging for time-domain simulations because of the different geometrical scales involved: The full length of a spiral arm is around $L_{S}=400 \mathrm{~mm}$, which represents $\sim 1600$ times the arm width or the substrate thickness, respectively. 


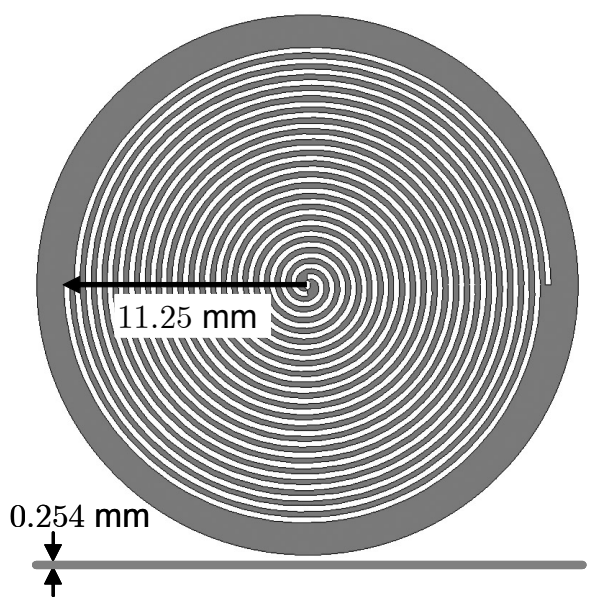

Figure 1: Top and side views of the spiral showing the two metallic arms (white) and the extent of the substrate (gray).

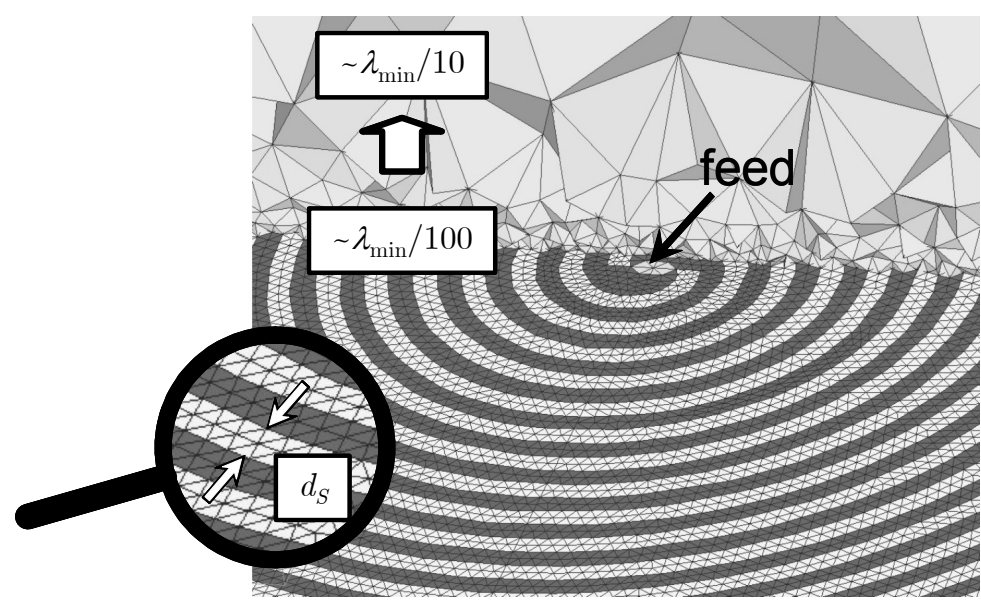

Figure 2: View of the discretized spiral. The image shows the triangulation of the spiral and part of the tetrahedral mesh.

\section{c) The Discretized Spiral Model}

A tetrahedral volume mesh and the associated triangulation of the surfaces, as commonly used in FVTD, is well suited for the modeling of a spiral structure for two main reasons (Figure 2). First, the triangulation of the antenna plane ensures a precise modeling of the spiral arms. Second, an inhomogeneous tetrahedral mesh permits to adapt locally the cell size to the dimensions of the fine structures that need to be resolved (here both, the fine spiral arms and the thin substrate). As illustrated in Figure 2 for the discretized model, the linear size of the tetrahedrons grows quickly from $\sim \lambda_{\min } / 100$ in the close proximity of the spiral to $\sim \lambda_{\min } / 10$ in freespace, where $\lambda_{\min }$ is the smallest wavelength of interest. This inhomogeneity of the mesh keeps the total number of cells in the model at a reasonable level. The spatial inhomogeneity of the mesh is combined with an inhomogeneous temporal discretization, implemented in the form of local time steps following the scheme demonstrated in [3].

In practical spiral antenna designs, a broadband balun is used for balanced feeding of the radiating spiral, with nearly constant input impedance over the desired frequency band. In the present simulations, a simple port consisting of 8 triangles is defined at the apex of the spiral. Both harmonic (at $8 \mathrm{GHz}$ ) and broadband (1$20 \mathrm{GHz}$ Gaussian pulse) excitations at the port have been applied in this study. The simulation of a practical balun has been performed separately and results have been presented in [4]. Inside the operation band of the spiral, the balun mostly determines the return loss.

\section{d) Convergence and Validation}

The side length of the triangles on the spiral plane corresponds to half the line width $d_{S}$ (magnified view in Figure 2). This fine discretization is necessary for the simulation to converge. Validation of the simulation results is provided through comparison with measurements of a cavity-backed spiral antenna. Simulation results obtained from a model that includes the substrate, the balun and the absorber-loaded cavity will be presented in future work.

It should be noted, that the strong inhomogeneity of the tetrahedral mesh permits to save computer memory. Despite a large number of triangles ( $>10 \mathrm{k}$ triangles per arm) on the spiral surface, a spiral model requires less than $250 \mathrm{MB}$ computer memory ( $\sim 400 \mathrm{k}$ tetrahedrons). Large simulation times however are necessary to allow the wave to propagate along the full length of the spiral arms and to be reflected.

\section{Results - Substrate Effects}

The effect of the substrate on the radiation characteristics of the spiral is observed through the current distribution on the spiral arms. The current densities on the spiral obtained through FVTD simulations with four different relative permittivities of the substrate are displayed in Figure 3. The presence of the substrate shortens the wavelength of the waves propagating along the arms of the spiral. The figure demonstrates the location of the classically defined active region where the radiation process is taking place on a spiral antenna: The active region is an annular band where currents on adjacent arms of the spiral are nearly in phase, which occurs for a 


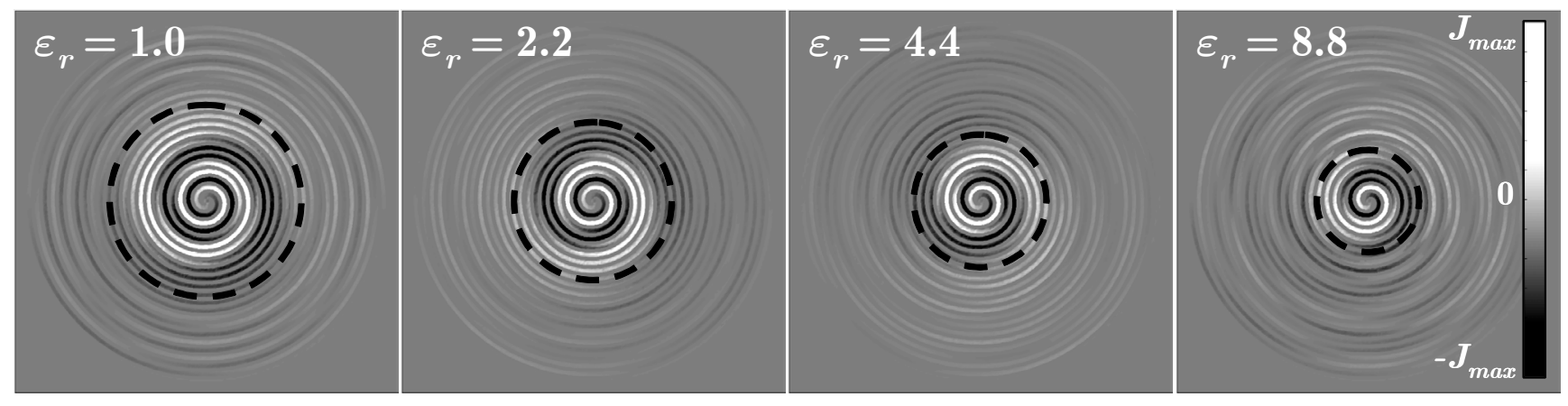

Figure 3: Simulated instantaneous current density distribution on the spiral at $8 \mathrm{GHz}$ for four substrate permittivities $\varepsilon_{r}$. The pictures also show as dashed circles the estimated radius of the active region.

radius nearly equal to $r_{a} \cong \lambda_{\text {eff }} / 2 \pi$. A dependence of the effective wavelength $\lambda_{\text {eff }}$ on the relative permittivity $\varepsilon_{r}$ of the spiral substrate is clearly visible in the plots.

The effective area is characterized more precisely from a 1D representation of the current distribution along the arms of the spiral. Figure 4 shows the envelope as well as an instantaneous current density along one arm of the spiral for harmonic excitation at $8 \mathrm{GHz}$. Two domains are visible on the graph: On the inner windings of the spiral (up to around 6 turns), the current density takes the form of a damped sinusoid. The active region of the spiral is located just before the end of this domain, where the effective wavelength $\lambda_{\text {eff }}$ is nearly equal to a full spiral turn $\Delta L$. Past the active region, on the outer turns of the spiral, the current distribution exhibits a nonsinusoidal standing-wave characteristic shaped by reflections at the end of the arms and by crosstalk between the windings.

The effective permittivity for propagation of currents on the spiral antenna as a function of the relative permittivity $\varepsilon_{r}$ of the substrate is presented in Figure 5 . The uncertainty grows with increasing $\varepsilon_{r}$ because of the nonlinear dependence $\lambda_{\text {eff }}\left(\varepsilon_{\text {eff }}\right)=\lambda_{0} / \sqrt{\varepsilon_{\text {eff }}}$. For comparison, the effective permittivity of corresponding coplanar strips (CPS) is represented in the same graph. It is noted that the field distribution of two adjacent arms of the spiral (with e.g. currents in phase on two adjacent lines in the active region) does not correspond to that of a balanced CPS. The $\varepsilon_{e f f}$ dependence obtained in case of the spiral corresponds to a value that would occur for a CPS with wider coplanar strips. The dependence of the effective permittivity on the substrate permittivity for the spiral is confirmed by observation of the circular polarization bandwidths and impedance obtained from the simulations.

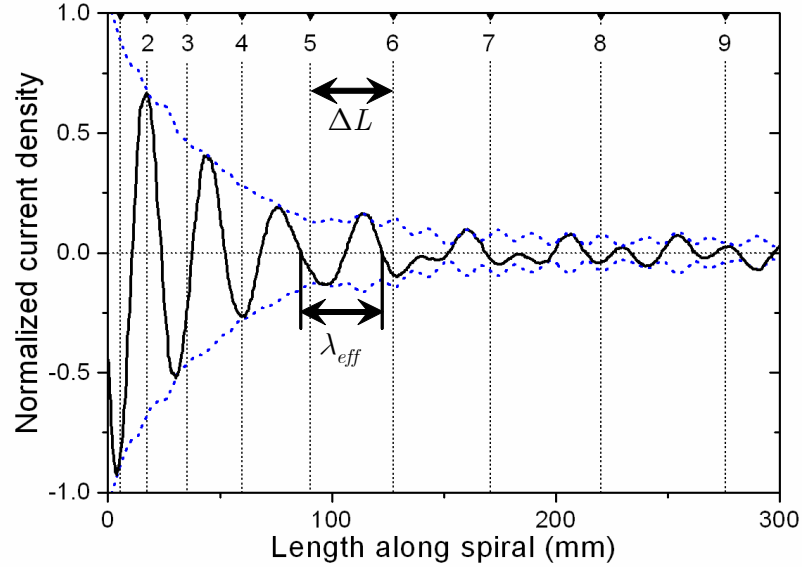

Figure 4: Instantaneous current density along the length of a spiral arm at $8 \mathrm{GHz}$ (solid line). Also plotted is the envelope (dotted lines). The upper scale indicates the turns on the spiral. The substrate has a relative permittivity $\varepsilon_{r}=2.2$.

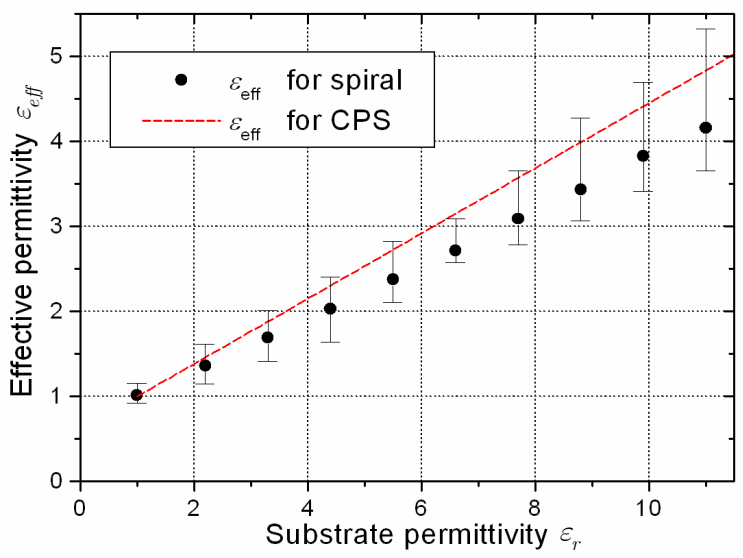

Figure 5: Estimated effective permittivity of the propagation of waves on the spiral antenna. The error bar indicates uncertainty in the active region, caused mainly by the influence of reflected waves. Also shown for comparison is the $\varepsilon_{\text {eff }}$ of a coplanar strip line (CPS) with similar dimensions $(W=0.25 \mathrm{~mm}$, gap $=0.25 \mathrm{~mm})$. 


\section{Conformal Spiral Antenna}

The simulation of spiral antennas has also been performed in conformal geometries for a similar design. Figure 6 shows examples of spirals on spherical and cylindrical substrates (radii of curvature of $18 \mathrm{~mm}$, thickness of substrate: $0.254 \mathrm{~mm}$ ). The computational cost of the FVTD simulations is identical to that of corresponding planar geometries. The shape of the supporting surface influences the radiation characteristics in several ways (Figure 7). For the spherical geometry, the effect is mostly visible in the front-to-back ratio of the radiation patterns. The directionality is clearly improved as it is the case in conical arrangements which share a similar axial symmetric geometry. For the cylindrical geometry an increased axial ratio indicates a degradation of the circular polarization purity. In this case, a turn exhibits a saddle shape which affects the phase between the orthogonal field components in the far-field, resulting in the polarization alteration. The observed oscillations of the axial ratio are due to the frequency-dependent angular and radial location of the active radiating region.

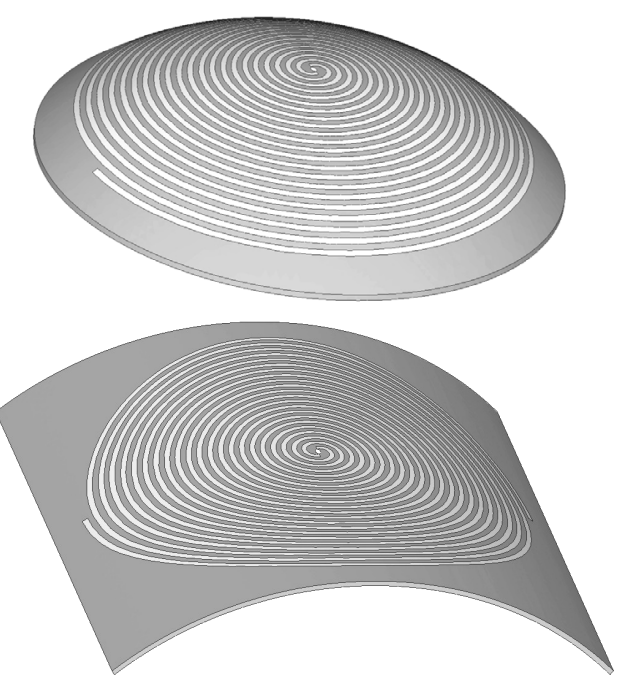

Figure 6: Conformal spiral geometries.
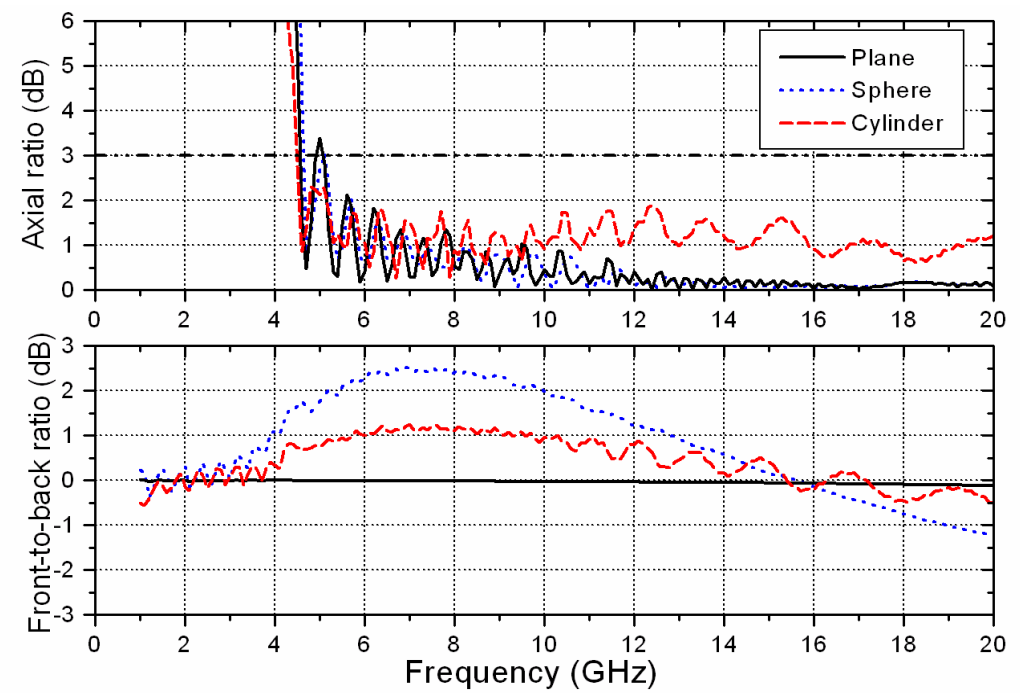

Figure 7: Polarization axial ratio and Front-to-back ratio as function of the frequency.

\section{Conclusion}

This paper has reported FVTD simulations of different configurations of Archimedean spiral antennas on thin-substrates. Such structures are very challenging for any simulation technique and serve to prove the advantages of the FVTD method as a very versatile EM simulation tool. The FVTD's unstructured mesh provides the geometrical flexibility needed to resolve spiral structures on thin substrates. The effect of the permittivity of the substrate on the current density and on the bandwidth of the spiral has been described by an effective permittivity characterizing the currents propagating on the spiral arms. The application of the FVTD technique to conformal spiral geometries has also been demonstrated.

\section{References}

[1] V. Shankar, A.H. Mohammadian, W.F. Hall, "A time-domain, finite-volume treatment for the Maxwell equations", Electromagnetics, Vol. 10, pp. 127-145, 1990

[2] P. Bonnet, X. Ferrieres, B.L. Michielsen, P. Klotz, J.L. Roumiguières, "Finite-volume time domain method", Chapt. 9 in "Time domain electromagnetics", ed. by S.M. Rao, Academic Press, San Diego, 1999

[3] C. Fumeaux, D. Baumann, P. Leuchtmann, R. Vahldieck, "A generalized local time-step scheme for efficient FVTD simulations in strongly inhomogeneous meshes", IEEE Trans. Microwave Theory Tech., Vol. MTT-52, pp. 1067-1076, March 2004

[4] D. Baumann, C. Fumeaux, P. Leuchtmann, R. Vahldieck, "Generalized-scattering-matrix extraction using the finitevolume time-domain (FVTD) method", IEEE MTT-S Int. Microwave Symp. Dig., pp. 1701-1704, June 2004 